\title{
The Clinical Efficacy and Safety of Neratinib in Combination with Capecitabine for the Treatment of Adult Patients with Advanced or Metastatic HER2-Positive Breast Cancer
}

\author{
Giovanna Chilà (iD* \\ Vincenzo Guarini* \\ Danilo Galizia \\ Elena Geuna \\ Filippo Montemurro ii \\ Multidisciplinary Oncology Outpatient \\ Clinic, Candiolo Cancer Institute, FPO- \\ IRCCS, Candiolo, 10060, Italy \\ *These authors contributed equally to \\ this work
}

\begin{abstract}
Human epidermal growth factor receptor 2 (HER2) positive cancers account for $15-20 \%$ of all breast tumors. Several drugs have been approved in the metastatic setting, including monoclonal antibodies, tyrosine kinase inhibitors (TKI) and, more recently, antibody-drug conjugates. Neratinib is a pan-HER, irreversible TKI with potent preclinical activity against trastuzumab-resistant breast cancer models. Based on Phase I and II clinical trials, the combination of neratinib plus capecitabine was compared to lapatinib and capecitabine, an established regimen for trastuzumab-resistant disease, in the randomized, Phase III NALA trial. In this trial, neratinib yielded increased progression-free survival, response duration and a benefit in time to intervention for CNS progression. However, there was no overall survival benefit, no increase in overall response rate and no improvement in QoL. The most frequent adverse event in the neratinib arm was diarrhea, which was manageable with prophylactic treatment with loperamide. Conclusion: Neratinib is a valuable addition to the therapeutic armamentarium to treat metastatic, HER2-positive breast cancer. The current positioning of the combination of neratinib and capecitabine based on the results of the NALA trial needs to consider the rapidly evolving scenario due to the recent introduction of new drugs, like the pure-HER2 TKI tucatinib and the antibody drug-conjugate trastuzumabderuxtecan.
\end{abstract}

Keywords: neratinib, tyrosine kinase inhibitors, breast cancer, HER2, metastasis, randomized clinical trial

\section{Introduction}

Breast cancer is the most common malignancy in women (approximately 30\% of total), where it represents the first cause of cancer-related death. ${ }^{1}$

From a biological point of view, breast cancer can be classified into molecularly defined subtypes, each one having different characteristics, clinical behavior and response to specific anticancer treatments. ${ }^{2,3}$

Among them, human epidermal growth factor receptor 2 (HER2) positive cancers account for $15-20 \%$ of all breast tumours. ${ }^{4}$

They are usually more aggressive, associated with a worse prognosis (especially if compared to hormone receptor (HR) positive/HER2 negative ones), often metastatic at diagnosis, and with a particular propensity to metastasize to the central nervous system.
Correspondence: Filippo Montemurro Multidisciplinary Oncology Outpatient Clinic, Candiolo Cancer Institute, FPOIRCCS, Trada Provinciale 142, Km 3.94 Candiolo, 10060, Italy

Tel +390119933250

$\mathrm{Fax}+390119933275$

Email filippo.montemurro@ircc.it 
In the last twenty years, since the introduction of trastuzumab, the first HER2-directed monoclonal antibody, the natural history of HER2 positive breast cancer has improved considerably. ${ }^{5}$

Fueled by further exploiting the therapeutic potential of HER2 targeting and to overcome pharmacological resistance, the armamentarium of new drugs is in constant expansion, resulting in significant improvements in the outlook of women bearing this subset of breast cancer.

\section{Standard Treatments}

In women with a diagnosis of trastuzumab-sensitive, advanced, HER2-positive breast cancer, the first line of treatment of choice is the association of taxane, pertuzumab and trastuzumab, based on the CLEOPATRA trial and PERUSE trial. ${ }^{6,7}$

For all patients who obtain at least the stability of the disease or even a response, either partial cor complete, maintenance therapy with pertuzumab and trastuzumab and, for those with hormone-receptor (HR)-positive disease, endocrine therapy is recommended. ${ }^{8}$

Two other very influential, randomized trials, EMILIA and TH3RESA, established the antibody-drug conjugate trastuzumab-emtansine (T-DM1) as the standard treatment for patients with trastuzumab and taxane, and trastuzumab and lapatinib-resistant disease, respectively. ${ }^{9-11}$

While first- and second-line treatments are quite established, based on the aforementioned randomized trials, there is currently no standard treatment for patients who are resistant to both trastuzumab/pertuzumab, and T-DM1. Rather, based on clinical trials conducted in the past, there are multiple therapeutic options, with none of them standing out as the preferred. ${ }^{11}$

\section{Neratinib}

Neratinib is a 4-anilino-3-cyano quinoline derivative and it is formulated in tablets as neratinib maleate. ${ }^{12}$ Neratinib irreversibly inhibits both HER1 and HER2, which, together with HER3 and HER4, constitute the HERfamily of transmembrane tyrosine kinase receptors. HER receptors are composed of two extracellular ligand-binding domains, a transmembrane domain, and an intracellular tyrosine kinase domain (except HER3), ${ }^{13}$ This family plays a fundamental role in several physiological cell processes and, when functionally altered like in the case of HER2-amplification or HER1/EGFR mutation, has a causative role in several human cancers. Because HERfamily members can either homo- or heterodimerize,
HER3 does not seem to have a strong tyrosine-kinase activity and the role of HER4 in cancer is still unknown, neratinib, like other similar compounds, is often referred as a pan-HER inhibitor.

Differently from HER1/EGFR and HER3, whose steric conformation changes upon ligand binding, HER2 has an "always open" conformation and, when overexpressed, forms homo and heterodimers with the other members of the family. Homo and, most importantly, heterodimerization activates the tyrosine-kinase activity of HERreceptors, triggering downstream signaling by the RasRaf-MAPK and the PI3K/AKT pathways, ${ }^{14}$ Neratinib forms a covalent binding with the cysteine residue (Cys773 in HER1 and Cys805 in HER2), located in the adenosine triphosphate protein (ATP) binding pocket of the receptor itself, thus determining the inhibition of the receptor kinase activity. Moreover, neratinib reduces the phosphorylation of the retinoblastoma protein, repressing cyclin D1 expression, and increases p27 protein levels, with the effect of blocking the cell cycle in the G1/S phase, resulting in the inhibition of cell proliferation and survival. ${ }^{15}$

In murine xenograft models, neratinib consistently inhibits the growth of HER2-dependent tumours, with no significant antitumor activity in low-HER2-expressing cell lines. ${ }^{16}$ Its efficacy has also been found in cell lines bearing HER2 somatic mutations (even if HER2 is not amplified), confirming the capacity of neratinib of overcoming possible resistance towards other anti-HER2 treatments. ${ }^{17}$

\section{Phase I Trials with Neratinib}

The first phase I trial with neratinib was published in $2009 .{ }^{18}$ It was an open-label study that enrolled 72 patients with HER2 or HER1/EGFR positive solid tumors ( $40 \%$ of them had breast cancer). Neratinib was administered as a single agent at escalating doses (from 40 to $500 \mathrm{mg}$ dose range), followed by one week of observation, and then once daily continuously.

This study focused on establishing the dose-limiting toxicity (DLT), maximum tolerated dose (MTD), pharmacokinetic profile and preliminary antitumor activity of neratinib. DLT was grade 3 diarrhea, experienced by one patient at the dose of $180 \mathrm{mg}$ daily and by four patients at the dose of $400 \mathrm{mg}$ daily. The MTD was therefore determined to be $320 \mathrm{mg}$ daily. All patients had adverse events, the most common being diarrhea (88\%) which was grade $3-4$ in $32 \%$ of patients. Nausea (64\%), fatigue $(63 \%)$, vomiting (50\%) and anorexia (40\%) were other frequent 
adverse events. Among these, only fatigue and vomiting reached grade 3 or 4 in a minority of patients ( $4 \%$ for both side effects). Overall, $31 \%$ of patients needed dose reductions, and (18\%) discontinued treatment because of toxicity. Diarrhea was the main cause of dose reductions and of temporary or definitive treatment discontinuation, which occurred most frequently during the first cycle.

Absorption of neratinib was relatively slow, with a median tmax of 3 to 6.5 hours. The steady state Cmax and the area under the curve (AUC) of neratinib increased with increasing dose in a nonlinear, reaching a plateau between 320- and 400-mg doses. The mean accumulation ratio was 1.14 after repeated doses of neratinib $240 \mathrm{mg}$ daily, indicating no major accumulation of the drug and the mean elimination half-life after a 240 dose of neratinib with food was 14 hours.

In the subset of 25 breast cancer patients who were available for tumor response, $8(32 \%)$, most of whom bearing histochemically HER2 3+ tumors, achieved at least a partial response (PR). The median duration of response (mDOR) was 4.8 months (95\% CI, 1.9-9.5) and the median progression-free survival (mPFS) was 3.6 months (95\% CI, $1.7-5.6)$.

Another phase I trial enrolled 21 Japanese patients with solid tumors, only three of whom had breast cancer. This was a dose-escalation study that assessed the safety, DLT, MTD, antitumor activity and pharmacokinetics of neratinib in this population.

Diarrhea and anorexia were the reported DLTs for $40 \%$ of patients at a dose of $320 \mathrm{mg}$. The MTD was then determined to be $240 \mathrm{mg}$. The most frequent adverse events were diarrhea $(95 \%$, which was at least grade 3 in $10 \%$ of patients), fatigue $(67 \%)$, nausea and abdominal pain $(43 \%)$. Two of the three patients with breast cancer (both pretreated with trastuzumab) had a partial response, and the duration of response was 16.1 and 32.3 weeks, respectively. $^{19}$

\section{Phase II Trials with Neratinib}

As a single agent, neratinib, at a dose of $240 \mathrm{mg}$ daily, was tested in a phase II trial that included 136 patients with HER2 positive breast cancer. ${ }^{20}$

The study enrolled two cohorts of patients, one pretreated with trastuzumab-based therapy (66 patients) and the other trastuzumab-naive (70 patients). The study met its primary endpoint, which was a 16-week PFS of more than $30 \%$, both in trastuzumab-pretreated (59\%) and in trastuzumab-naive patients (78\%). mPFS was 22.3 and
39.6 weeks, and Overall Response Rate (ORR) was 24\% and $56 \%$, for trastuzumab-pretreated, and trastuzumabnaive patients, respectively. Diarrhea was the main adverse event, with an all-grade incidence of $93 \%$ overall $(97 \%$ and $87 \%$ in the first and second cohort, respectively), requiring dose reductions in $29 \%$ and $4 \%$ of the patients in the first and second cohort, respectively. Yet only one patient had to discontinue treatment because of diarrhea. Other significant side effects were nausea (36\%), vomiting (31\%) and fatigue (24\%).

The safety and efficacy of neratinib were compared to that of capecitabine plus lapatinib in a non-inferiority phase II trial. $^{21}$ The study enrolled 233 patients who had to be previously treated with a taxane plus trastuzumab. Neratinib was administered orally at a daily dose of $240 \mathrm{mg}$ continuously, lapatinib at a dose of $1250 \mathrm{mg}$ daily continuously and capecitabine at a dose of $1000 \mathrm{mg} / \mathrm{m}^{2}$ bis in die (BID) on days 1-14 of each 21day cycle. In terms of the primary study end-point, which was PFS and mPFS, neratinib failed to show non inferiority, with a mPFS of 4.5 months and 6.8 months for neratinib and capecitabine plus lapatinib, respectively.

Neratinib showed inferior performance also regarding the secondary endpoints of Overall Survival (OS) (19.7 months vs 23.6 months), ORR (29\% vs 41\%, p 0.067) and Clinical Benefit Rate (CBR, 44\% vs 64\%, p 0.003). Despite failing to show non inferiority, the clinical activity of neratinib as a single agent was confirmed in this study. Adverse events for both arms were mainly gastrointestinal, but neratinib was associated with fewer dose reductions ( $20 \%$ vs $53 \%$, respectively), dose delays (32\% vs $74 \%$ ) and treatment discontinuations ( $6 \%$ vs $17 \%)$.

Building on these results, a phase I/II trial, published by Saura et al in $2014,{ }^{22}$ explored the combination of neratinib and capecitabine.

The first part of this clinical trial was a dose finding study of neratinib and capecitabine in patients with different solid tumors. The MTD of the combination was $240 \mathrm{mg}$ daily for neratinib continuously and $750 \mathrm{mg} / \mathrm{m}^{2}$ BID for capecitabine administered on days 1 to 14 of each 21-day cycle. In the second part of the study, these doses of the two compounds were administered to 72 HER2positive, advanced breast cancer patients previously treated with trastuzumab. Of these, 65 were lapatinib-naive. The ORR and CBR were $64 \%$ and $72 \%$, respectively, in lapatinib-naive, and $57 \%$ and $71 \%$, respectively, in lapatinib-treated patients. PFS was 40.3 vs 35.9 weeks, for lapatinib-naive and -treated, respectively. 


\section{The NALA Trial}

Based on the encouraging phase II results, a randomized, phase III trial comparing neratinib plus capecitabine vs lapatinib plus capecitabine was launched in $2013 .^{23}$

The NALA trial enrolled a total of 628 patients from 28 different countries, all of whom pretreated with at least 2 anti-HER2 therapeutic lines for the advanced setting. Patients with asymptomatic or stable central nervous system (CNS) disease were eligible.

Patients were assigned in a 1:1 ratio to receive neratinib $240 \mathrm{mg}$ daily plus capecitabine $750 \mathrm{mg} / \mathrm{m}^{2}$ BID 1-14/ 21 or lapatinib $1250 \mathrm{mg}$ daily plus capecitabine $1000 \mathrm{mg} /$ $\mathrm{m}^{2}$ BID 1-14/21.

Coprimary endpoints of the study were PFS and OS, while the secondary endpoint was time to intervention for symptomatic CNS disease.

After a median follow-up of 29.9 months, the combination of neratinib plus capecitabine yielded a statistically significant, $24 \%$ decrease in risk of progression or death (HR 0.76, 95\% CI, 0.63-0.93, p 0.0059). Interestingly, the PFS curves overlapped during the first 24 weeks, resulting in similar mPFS (5.6 vs 5.5 months, for neratinib and lapatinib, respectively) and subsequently started to separate in favor of neratinib. This resulted in a gain of 2.2 months in the 24-month restricted mean PFS (8.8 vs 6.6 months for neratinib and lapatinib, respectively). The benefit was larger in patients with non-visceral disease (HR 0.44, 95\% CI, 0.26-0.73, p 0.007) and HR-negative tumors (HR 0.42, 95\% CI, 0.31-0.47, p < 0.001).

Although there was a trend towards a $12 \%$ reduction in the risk of death, neratinib failed to significantly improve OS. Another intriguing finding of this trial was that, despite similar ORR (32.8\% vs $26.7 \%)$, the mDOR, which was 8.5 vs 5.6 months (HR $0.5,95 \%$ CI, $0.33-$ $0.74, \mathrm{p} 0.0004$ ) for neratinib and lapatinib, respectively.

The combination with neratinib also showed interesting results regarding the risk of CNS progression. In fact, the overall cumulative incidence of intervention for CNS progression during the trial was $22.8 \%(95 \% \mathrm{CI}, 15.5 \%$ $30.9 \%$ ) vs $29.2 \%$ (95\% CI, $22.5 \%-36.1 \%$ ) in the neratinib and lapatinib arm, respectively.

The safety profile of neratinib was consistent with previous findings from the phase I and II trials. The most common adverse event was diarrhea of grade 3 (24.4\% with neratinib vs $12.5 \%$ with lapatinib), occurring mostly during the first cycle, causing a dose reduction of neratinib in $5.3 \%$ and its permanent discontinuation in $2.6 \%$ of patients. However, corresponding rates in the lapatinib arm were similar ( $4.2 \%$ and $2.3 \%$, respectively)

Other frequent all grade adverse events were Hand-foot syndrome (HFS) $(45.9 \%$ vs $56.3 \%)$, nausea $(53.1 \%$ vs $42.4 \%)$, vomiting $(45.5 \%$ vs $31.2 \%)$ and fatigue $(34.3 \%$ vs $31.2 \%)$. Finally, both discontinuation rates due to safety issues and Health Related Quality of Life were not significantly different in the two groups of patients.

Considering these results, the combination of neratinib plus capecitabine was approved by the Food and Drug Administration (FDA) in February 2020 in patients with HER2 positive metastatic breast cancer who had already received at least two therapeutic lines in this setting.

\section{Neratinib-Related Diarrhea}

As already discussed, neratinib toxicity mainly involves the gastrointestinal tract.

Diarrhea is the most common adverse event, followed by nausea, vomiting, abdominal pain and anorexia. Based on several studies, it is likely that neratinib-related diarrhea is caused by HER1/EGFR inhibition. ${ }^{24,25}$

Tao et al published a systematic review including 23 studies for a total of 4896 patients treated with neratinib alone or in combination with other anticancer agents (temsirolimus, paclitaxel, capecitabine, vinorelbine, and trastuzumab). ${ }^{26}$

Diarrhea was confirmed to be the main toxicity, particularly when neratinib was administered in combination with other drugs. Neratinib plus vinorelbine, for example, was associated with the highest rate of all grade diarrhea $(95.1 \%)$, while the combination of neratinib and paclitaxel yielded 32.1\% Grade (G) 3-4 diarrhea. Neratinib monotherapy, on the contrary, had a more favorable profile ( $83.9 \%$ for all grades, and $25.1 \%$ for grade $3-4$ diarrhea).

Due to significant patient discomfort, possible associated complications (for example, important dehydration and electrolyte abnormalities), and the risk of treatment discontinuation, prophylactic loperamide is an important component of treatment with this agent. A $61.3 \%$ reduction of all grade diarrhea for patients treated with neratinib in monotherapy who received prophylactic therapy has been reported and supports this practice. Loperamide should be started early and administered daily during the first cycle, and subsequently adjusted as needed. ${ }^{27}$ Clinically valuable information regarding the management of neratinib-related diarrhea has been recently provided from the extended adjuvant setting. In women who have completed one year of adjuvant trastuzumab and are still at 
risk for subsequent relapse, neratinib (240 $\mathrm{mg}$ daily) is currently approved based on the results of the EXTENET trial. $^{28,29}$ Recently, the CONTROL study evaluated different proactive pharmacological interventions to limit diarrhea, which in the EXTENET trial, where no prophylaxis was protocol-defined, reached grade 3 or superior and caused permanent treatment discontinuation in $40 \%$ and $17 \%$ of the patients, respectively. ${ }^{30}$ Results of the CONTROL study show that serious diarrhea and permanent discontinuation of neratinib could be reduced by using prophylactic loperamide alone budesonide or colestipol (various combinations were tested in subsequent cohorts) or by a dose escalation approach. Starting from doses of 120 or $160 \mathrm{mg}$ daily and increasing to the full daily dose according to a predefined escalation plan, worst grade diarrhea was G3 and occurred in $15 \%$ of the patients. Furthermore, the permanent discontinuation rate of neratinib because of diarrhea was $3 \%$.

\section{Neratinib Activity in CNS Disease}

Brain metastases occur frequently in patients with HER2positive breast cancer, with incidences of $30-55 \%$ in those with metastatic disease. ${ }^{31,32}$

The high rate of CNS lesions in these patients is explained by the tropism of HER2-positive disease for CNS sites, the prolonged overall survival and the poor activity of anti-HER2-monoclonal antibody beyond the blood-brain barrier. $^{33-35}$

For these reasons, CNS disease represents, still today, a therapeutic challenge in this patient setting. Due to small dimensions and lower molecular weight, together with their mechanism of action, tyrosine kinase inhibitors (TKIs) have been pointed as potentially effective drugs in patients with CNS involvement. ${ }^{36}$

Indeed, encouraging results have been reported in the past with TKIs, both as single agents and in combination with chemotherapy, in women with HER2-positive breast cancer metastatic to the $\mathrm{CNS}^{37}$

The phase II trial NEfERT-T explored the use of paclitaxel $\left(80 \mathrm{mg} / \mathrm{m}^{2}\right.$ on days $1,8,15$ every 28 days) plus neratinib at a standard dose of $240 \mathrm{mg}$ orally daily or trastuzumab (4 mg/kg then $2 \mathrm{mg} / \mathrm{kg}$ weekly) as first-line treatment in women with HER2-positive metastatic breast cancer. $^{38}$

Women with CNS metastases could be enrolled, if they were asymptomatic after appropriate local treatments and free from steroids and/or anticonvulsant for at least 4 weeks before study entry. However, only a total of 18 patients with CNS involvement were enrolled. The study showed a mPFS of 12.9 months for both treatment arms (HR 1.02; 95\% CI, 0.81-1.27; p 0.89), with similar outcomes in all patient subgroups. Furthermore, ORR (74.8\% vs $77.6 \%$, p 0.52 ), CBR (88.4\% vs $85.2 \%$, p 0.24$)$ and the mDOR were also similar in both arms (HR 1.01; $95 \mathrm{CI}$, $0.78-1.32$, p 0.92).

Interestingly, CNS recurrence, which was a secondary study endpoint, was less frequent in patients in the neratinib arm (8.3\% vs 17.3\%; HR 0.48; 95\% CI, 0.29-0.79; $\mathrm{p}$ 0.002). The safety profile was consistent with that reported in all the other studies with neratinib: the most frequent G3 adverse event was diarrhea (30.4\% vs 3.8\%, $\mathrm{p}<0.001)$.

Another Phase II, multi-cohort clinical trial, TBCRC 022, evaluated neratinib efficacy in patients with CNS metastases progressing after prior lines of treatment, including surgery and/or radiation therapy. ${ }^{39}$ Of 40 patients receiving single-agent neratinib at the conventional dose of $240 \mathrm{mg}$ /day continuously, only three achieved a Partial Response (PR) (8\%). Despite these disappointing results, neratinib was further explored in combination with capecitabine at the dose of $750 \mathrm{mg} / \mathrm{m}^{2}$ BID $1-14 / 21$ in two further cohorts of this study, which were defined on the basis of the prior receipt of lapatinib.

The study showed a CNS overall response rate of $49 \%$ vs $33 \%$ and a mPFS of 5.5 vs 3.1 months, in lapatinibnaive and pre-treated patients, respectively.

All together, these results confirmed the activity of neratinib on CNS disease, pointing out the importance of chemotherapy in enhancing the efficacy of HER2 treatments, especially in refractory brain disease.

Further insights from the NALA trial were reported at the 2020 San Antonio Breast Cancer Symposium. ${ }^{23}$ A post-hoc analysis was conducted on patients who had asymptomatic and stable CNS disease at baseline (16.3\% of total, 51 and 50 patients in the neratinib and lapatinib arm, respectively), confirming a positive trend in terms of PFS in favor of neratinib (7.8 months vs 5.5 months). Moreover, this analysis, similarly to the main analysis, revealed a lower number of interventions for CNS disease and a lower percentage of patients treated for brain metastases after 12 months (25.5\% vs $36 \%$, respectively). Neratinib plus capecitabine was also associated with prolonged CNS-PFS (12.4 months vs 8.3 months). These data provide further ground to consider neratinib and capecitabine as a valuable option in patients with HER2 positive metastatic breast cancer and CNS involvement. 


\section{Discussion}

The NALA trial is one of the recently published clinical trials contributing to redesigning the current therapeutic algorithm for HER2-positive metastatic breast cancer. Since the introduction of lapatinib, and, more recently, pertuzumab and T-DM1, this algorithm has remained, basically, unchanged for almost a decade. ${ }^{11}$ Therefore, the obvious question is how the NALA results fill the current gaps and answer the open questions in this disease setting. The last three years have seen an unprecedented showcase of new drugs for the treatment of HER2 positive disease. The tyrosine kinase inhibitors tucatinib and pyrotinib, ${ }^{40,41}$ the monoclonal antibody margetuximab and the newer antibody-drug conjugates trastuzumabderuxtecan and trastuzumab-duocarmazine have already proven their worth in phase II or phase III trials in patients progressing on prior lines of treatment. ${ }^{42-44}$ Research is now focusing on evaluating their role in earlier lines for metastatic disease, and even in the operable setting. Thus, the clinical positioning of neratinib must be considered in a rapidly changing scenario. The comparison made in the NALA was aimed at answering the simple question:

Is neratinib a better TKI than lapatinib, when added to capecitabine in women with HER2-positive advanced breast cancer, who are resistant to monoclonal antibodybased therapy?

The results provide evidence that this may be the case. Neratinib yielded increased response duration, which likely supports the observed PFS advantage beyond the initial six months of treatment. The benefit in time to intervention for CNS progression, although small, is clinically significant, due to the challenges related to this clinical situation. However, there is no overall survival benefit, no increase in overall response rate, and no improvement in Qol parameters. To try to put these results into the context, it must be said that the clinical scenario has changed significantly since this trial enrolled the first patient in May 2013. In fact, the study was designed and partially conducted before the widespread diffusion of pertuzumab and T-DM1 in first and second line, respectively. At that time, lapatinib and capecitabine was one of the possible standards for patients with trastuzumab-resistant, HER2-positive advanced breast cancer. The alternative option was the continuation of trastuzumab, with a change in the companion chemotherapy drug. The accrual was completed in July 2017, resulting in a profile of the enrolled patients that only partially reflects that of patients who may be eligible for a TKI at the present time. In fact, prior exposure to trastuzumab, pertuzumab and T-DM1, occurred in only one third of the enrolled patients, with $40 \%$ of patients previously exposed to trastuzumab only. While this does not affect the internal validity and the interpretation of results, it inevitably calls into question indirect comparisons. The first, and more obvious, is with the pure HER2-TKI Tucatinib. ${ }^{40}$ The HER2CLIMB trial tested the addition of this compound to treatment with trastuzumab and capecitabine in women with HER2-positive, metastatic breast cancer who were resistant to trastuzumab, pertuzumab and T-DM1. Accrual was completed between February 2016 and May 2019, thus better reflecting the contemporary context, with almost $100 \%$ of patients receiving prior trastuzumab, pertuzumab and T-DM1 (exposure to pertuzumab and T-DM1 was protocolmandated). Tucatinib proved to be superior in all the planned outcomes, including PFS, OS, ORR and Quality of life. ${ }^{45}$ Furthermore, in a pre-planned analysis of a large stratum of patients with CNS involvement at study entry, clinically relevant improvements in outcomes were also observed. ${ }^{46}$ The hype around the HER2-climb results has led to the suggestion that this combination may be preferable to the current standard (T-DM1), in patients progressing on trastuzumab/pertuzumab, in particular if they have CNS involvement. One weakness of this study, at least in our opinion, was, differently from the NALA trial, the lack of a TKI in the comparison arm. In fact, phase II clinical trials conducted in the past have suggested a meaningful clinical activity of lapatinib and capecitabine in patients with CNS metastases from HER2-positive breast cancer. $^{47,48}$ Although lack of phase III data is a limitation to the clinical applicability of these results, guidelines have suggested lapatinib-based therapy as an option in patients with this pattern of metastatic disease. ${ }^{49}$ In practical terms, in the subset of patients with CNS involvement, the tucatinib-containing arm has been compared with a regimen that non all the medical oncologist would have opted for in the clinical practice in similar patients. Notably, prior exposure to lapatinib was allowed if it had occurred more than twelve months prior to enrolment. As a result, the HER2CLIMB population was, practically, lapatinib-naive, with only $6 \%$ of patients having received this drug as prior treatment. This begs the question of the adequacy of the control 
Table I Summary of Comparator Arms in Randomized Clinical Trials Evaluating New Agents in the Treatment of TrastuzumabResistant, HER2 Positive Advanced Breast Cancer

\begin{tabular}{|c|c|c|c|c|c|c|c|}
\hline Trial & Treatment & $\begin{array}{l}\text { Exposure to } \\
\text { Pertuzumab (\%) }\end{array}$ & $\begin{array}{l}\text { Exposure to } \\
\text { T-DMI (\%) }\end{array}$ & $\begin{array}{l}\text { Prior Lines } \\
\text { for MBC }\end{array}$ & mPFS & mos & $\begin{array}{l}\text { ORR } \\
\text { (\%) }\end{array}$ \\
\hline HER2CLIMB ${ }^{40}$ & Trastuzumab plus Capecitabine & 99.4 & 100 & Median $3(|-| 3)$ & 5.6 & 17.4 & 22.8 \\
\hline NALA $^{23}$ & Lapatinib plus Capecitabine & 44.0 & 57 & 2 in $70 \%$ of pts & $\approx 5.8$ & $\approx 18$ & 26.7 \\
\hline SOPHIA ${ }^{42}$ & Trastuzumab plus CT & 99.6 & 91.5 & $\leq 2$ in $66 \%$ of pts & 4.9 & 19.8 & 16.0 \\
\hline EMILIA 9 & Lapatinib plus Capecitabine & 0 & 0 & $0-1$ in $60 \%$ od pts & 6.4 & 25.1 & 30.8 \\
\hline TH3RESA $* 10$ & Trastuzumab plus CT & 0 & 0 & $\geq 4$ in $61 \%$ of pts & 3.2 & 15.8 & 9.0 \\
\hline
\end{tabular}

Notes: *All patients had to have been previously exposed to both trastuzumab- and lapatinib-based therapy.

Abbreviations: MBC, metastatic breast cancer; N, number; mPFS, median progression-free survival in months; mOS, median overall survival in months; ORR, overall response rate; $\mathrm{CT}$, chemotherapy.

arm in the HER2CLIMB trial. While there is no methodologically sound answer to this question, we noted that the performance of trastuzumab and capecitabine in the HER2CLIMB trial, seems to be in line with other comparators in randomized trials, both in the overall populations, (Table 1), and in studies focusing on patients with CNS metastases using lapatinib or neratinib (Table 2). Obviously, indirect comparisons need to be interpreted with caution. Yet, these are inevitable in clinical decision making when different options are available. In this case, they are reassuring that the results of tucatinib may not be due to the suboptimal performance of the comparison arm. Thus, in the context of patients who have failed trastuzumab/pertuzumab and T-DM1, there should be no issue of whether neratinib is a better companion than lapatinib for capecitabine (and trastuzumab), if tucatinib is available. To introduce a further level of complication, other antibody-drug conjugates like Trastuzumab-Deruxtecan, that has shown remarkable phase II results, will soon provide phase III results that will likely be practice changing.

Table 2 Overview of Drugs with Activity in CNS Metastases from HER2-Positive Breast Cancer

\begin{tabular}{|c|c|c|c|c|c|c|}
\hline Author (Year) & Drugs & Setting (CNS Status) & $\mathbf{N}$ & IC-ORR (\%) & mPFS & mos \\
\hline $\operatorname{Lin}(2009)^{47}$ & Lapatinib plus Capecitabine & Phase II, prior RT, progressive & 50 & $20^{\mathrm{a}}$ & 3.6 & 6.4 \\
\hline Bachelot $(2013)^{48}$ & Lapatinib plus Capecitabine & Phase II, no prior therapy & 45 & $57^{b}$ & 5.5 & 17 \\
\hline Freedman $(2019)^{52}$ & Neratinib plus Capecitabine & Phase II, progressive & 37 & $49^{f}$ & 5.5 & 13.3 \\
\hline \multirow[t]{2}{*}{ Saura $(2020)^{23}$} & Lapatinib plus Capecitabine & \multirow[t]{2}{*}{ Phase III, subgroup, asymptomatic ${ }^{d}$} & 50 & $15.4^{\mathrm{b}}$ & $5.5^{\mathrm{c}}$ & $15.4^{\mathrm{c}}$ \\
\hline & Neratinib + Capecitabine & & 51 & $26.3^{\mathrm{b}}$ & $7.8^{c}$ & $16.4^{\mathrm{c}}$ \\
\hline Montemurro $(2020)^{53}$ & T-DMI & Phase II, post hoc, asymptomatic ${ }^{d}$ & 126 & 42.9 & 5.5 & 18.7 \\
\hline Jerusalem $(2020)^{54}$ & T-DXT & Pase III, post hoc, asymptomatic ${ }^{d}$ & 24 & 58.3 & 18.1 & N.R. \\
\hline \multirow[t]{2}{*}{$\operatorname{Lin}(2020)^{46}$} & Tucatinib, Capecitabine, Trastuzumab & \multirow[t]{2}{*}{ Pase III, exploratory ${ }^{\mathrm{e}}$} & 198 & $47.3^{g}$ & 9.9 & I8. I \\
\hline & Capecitabine plus Trastuzumab & & 93 & $20.0^{\mathrm{g}}$ & 4.2 & 12 \\
\hline
\end{tabular}

Notes: ${ }^{\mathrm{a}} \geq 50 \%$ volumetric reduction. ${ }^{\mathrm{b}} \mathrm{RECIST}$ I.I criteria. ${ }^{\mathrm{c}}$ Restricted mean PFS ( 24 months) and OS (48 months). ${ }^{\mathrm{d}}$ With or without prior RT or local treatments. ${ }^{\mathrm{e}}$ Treated stable, treated progressing, untreated asymptomatic with or without low dose steroids/mannitol. ${ }^{\mathrm{f} C}$ Composite CNS response criteria (Volume+symptoms+steroid use). ORR by RANO criteria was $22 \% .{ }^{g}$ Active metastases

Abbreviations: CNS, central nervous system; IC-ORR, intracranial overall response rate; mPFS, median progression-free survival in months; mOS, median overall survival in months; T-DMI, trastuzumab emtansine; T-DXT, trastuzumab-deruxtecan.

HER, human epidermal growth factor receptor; HR, hormone receptor; TDMI, trastuzumab-emtansine; ATP, adenosine triphosphate protein; DLT, dose-limiting toxicities; MTD maximum tolerated dose; AUC, area under the curve; mDOR, median duration of response; mPFS, median progression free survival; PFS, progression free survival; ORR, objective response rate; BID, bis in die; OS, overall survival; CBR, clinical benefit rate; CNS, central nervous system; HFS, hand-foot syndrome; FDA, Food and Drug Administration; G, grade; TKIs, tyrosine kinase inhibitors; PR, partial response. 


\section{Conclusion}

Despite all the considerations regarding the actual impact of the NALA results outlined in the Discussion, neratinib remains a drug to be considered in the therapeutic armamentarium to treat HER2-positive disease for several reasons. The most obvious is that it is an active drug and HER2-positive disease may retain responsiveness beyond progression to different treatments. Furthermore, some peculiarities of neratinib that have emerged need to be explored in the context of precision oncology. For example, because HER2-amplified tumors may acquire HER2 mutations during clonal evolution under treatment pressure, a potent, irreversible pan-HER2 inhibitor may be a rational choice. ${ }^{50}$ Early results with neratinib in HER2mutated cancers provide grounds in this respect. ${ }^{51}$ Secondly, both the NALA in the metastatic setting and the EXTE-net trial in the extended adjuvant therapy setting show an interaction between the efficacy of neratinib and hormone-receptor coexpression. $^{28}$ In these tumors, neratinib may be an optimal companion of endocrine therapy to successfully disrupt crosstalk between the HER 2 and estrogen receptor pathways, which is responsible for drug resistance. ${ }^{8}$

\section{Acknowledgments}

FM and G.C. are supported by SEE-HER/PTCRC-Intra 2020 - Programme for Translational Clinical Research in Cancer" - (FPRC 5xmille 2017 Ministero Salute)

\section{Disclosure}

F.M. has received honoraria for participation in advisory boards from Roche, Eli Lilly, Novartis, Astra Zeneca, SeaGen, Pfizer and Pierre Fabre. The other authors report no conflicts of interest in this work.

\section{References}

1. Siegel RL, Miller KD, Fuchs HE, Jemal A. Cancer Statistics, 2021. CA Cancer J Clin. 2021;71(1):7-33. doi:10.3322/caac.21654

2. Perou CM, Sørlie T, Eisen MB, et al. Molecular portraits of human breast tumours. Nature. 2000;406(6797):747-752. doi:10.1038/ 35021093

3. Sørlie T, Perou CM, Tibshirani R, et al. Gene expression patterns of breast carcinomas distinguish tumor subclasses with clinical implications. Proc Natl Acad Sci U S A. 2001;98(19):10869-10874. doi:10.1073/pnas.191367098

4. Montemurro F, Scaltriti M. Biomarkers of drugs targeting HER-family signalling in cancer. J Pathol. 2014;232(2):219-229. doi:10.1002/ path.4269

5. Milani A, Montemurro F, Gioeni L, Aglietta M, Valabrega G. Role of trastuzumab in the management of HER2-positive metastatic breast cancer. Breast Cancer. 2010;2:93-109. doi:10.2147/BCTT.S6070

6. Baselga J, Cortés J, Kim S-B, et al. Pertuzumab plus trastuzumab plus docetaxel for metastatic breast cancer. $N$ Engl J Med. 2012;366 (2):109-119. doi:10.1056/NEJMoa1113216
7. Bachelot T, Ciruelos E, Schneeweiss A, et al. Preliminary safety and efficacy of first-line pertuzumab combined with trastuzumab and taxane therapy for HER2-positive locally recurrent or metastatic breast cancer (PERUSE). Ann Oncol. 2019;30(5):766-773. doi:10.1093/annonc/mdz061

8. Montemurro F, Di Cosimo S, Arpino G. Human epidermal growth factor receptor 2 (HER2)-positive and hormone receptor-positive breast cancer: new insights into molecular interactions and clinical implications. Ann Oncol. 2013;24(11):2715-2724. doi:10.1093/ annonc/mdt287

9. Verma S, Miles D, Gianni L, et al. Trastuzumab emtansine for HER2-positive advanced breast cancer. $N$ Engl J Med. 2012;367 (19):1783-1791. doi:10.1056/NEJMoa1209124

10. Krop IE, Kim S-B, González-Martín A, et al. Trastuzumab emtansine versus treatment of physician's choice for pretreated HER2-positive advanced breast cancer (TH3RESA): a randomised, open-label, Phase 3 trial. Lancet Oncol. 2014;15(7):689-699. doi:10.1016/ s1470-2045(14)70178-0

11. Giordano SH, Temin S, Davidson NE. Systemic therapy for patients with advanced human epidermal growth factor receptor 2-positive breast cancer: ASCO Clinical Practice Guideline Update Summary. J Oncol Pract. 2018;14(8):501-504. doi:10.1200/JOP.18.00290

12. Wissner A, Mansour TS. The development of HKI-272 and related compounds for the treatment of cancer. In: Archiv der Pharmazie; 2008;341(8):465-477. doi:10.1002/ardp.200800009.

13. Baselga J, Swain SM. Novel anticancer targets: revisiting ERBB2 and discovering ERBB3. Nat Rev Cancer. 2009;9(7):463-475. doi: $10.1038 / \mathrm{nrc} 2656$

14. Linggi B, Carpenter G. ErbB receptors: new insights on mechanisms and biology. Trends Cell Biol. 2006;16(12):649-656. doi:10.1016/j. tcb.2006.10.008

15. Rabindran SK, Discafani CM, Rosfjord EC, et al. Antitumor activity of HKI-272, an orally active, irreversible inhibitor of the HER-2 tyrosine kinase. Cancer Res. 2004;64(11):3958-3965. doi:10.1158/ 0008-5472.CAN-03-2868

16. Rabindran SK. Antitumor activity of HER-2 inhibitors. Cancer Lett. 2005;227(1):9-23. doi:10.1016/j.canlet.2004.11.015

17. Canonici A, Gijsen M, Mullooly M, et al. Neratinib overcomes trastuzumab resistance in HER2 amplified breast cancer. Oncotarget. 2013;4(10):1592-1605. doi:10.18632/oncotarget.1148

18. Wong -K-K, Fracasso PM, Bukowski RM, et al. A phase I study with neratinib (HKI-272), an irreversible pan ErbB receptor tyrosine kinase inhibitor, in patients with solid tumors. Clin Cancer Res. 2009;15(7):2552-2558. doi:10.1158/1078-0432.CCR-08-1978

19. Ito Y, Suenaga M, Hatake K, et al. Safety, efficacy and pharmacokinetics of neratinib (HKI-272) in Japanese patients with advanced solid tumors: a Phase 1 dose-escalation study. Jpn J Clin Oncol. 2012;42(4):278-286. doi:10.1093/jjco/hys012

20. Burstein HJ, Sun Y, Dirix LY, et al. Neratinib, an irreversible ErbB receptor tyrosine kinase inhibitor, in patients with advanced ErbB2-positive breast cancer. J Clin Oncol. 2010;28(8):1301-1307. doi:10.1200/JCO.2009.25.8707

21. Martin M, Bonneterre J, Geyer CE, et al. A phase two randomised trial of neratinib monotherapy versus lapatinib plus capecitabine combination therapy in patients with HER2+ advanced breast cancer. Eur J Cancer. 2013;49(18):3763-3772. doi:10.1016/j. ejca.2013.07.142

22. Saura C, Garcia-Saenz JA, Xu B, et al. Safety and efficacy of neratinib in combination with capecitabine in patients with metastatic human epidermal growth factor receptor 2-positive breast cancer. J Clin Oncol. 2014;32(32):3626-3633. doi:10.1200/JCO.2014.56.3809

23. Saura C, Oliveira M, Feng Y-H, et al. Neratinib plus capecitabine versus lapatinib plus capecitabine in HER2-Positive metastatic breast cancer previously treated with $\geq 2$ HER2-Directed Regimens: phase III NALA Trial. J Clin Oncol. 2020;38(27):3138-3149. doi:10.1200/ JCO.20.00147 
24. Opleta-Madsen K, Hardin J, Gall DG. Epidermal growth factor upregulates intestinal electrolyte and nutrient transport. $\mathrm{Am}$ J Physiol Gastrointest Liver Physiol. 1991;260(6):G807-G814. doi:10.1152/ajpgi.1991.260.6.g807

25. Goodlad RA, Raja KB, Peters TJ, Wright NA. Effects of urogastrone-epidermal growth factor on intestinal brush border enzymes and mitotic activity. Gut. 1991;32(9):994-998. doi:10.1136/gut.32.9.994

26. Tao Z, Li SX, Shen K, Zhao Y, Zeng H, Ma X. Safety and efficacy profile of neratinib: a systematic review and meta-analysis of 23 Prospective Clinical Trials. Clin Drug Investig. 2019;39(1):27-43. doi:10.1007/s40261-018-0719-0

27. Ustaris F, Saura C, Di Palma J, et al. Effective management and prevention of Neratinib-induced diarrhea. Am J Hematol. 2015;11(11).

28. Martin M, Holmes FA, Ejlertsen B, et al. Neratinib after trastuzumab-based adjuvant therapy in HER2-positive breast cancer (ExteNET): 5-year analysis of a randomised, double-blind, placebo-controlled, phase 3 trial. Lancet Oncol. 2017;18 (12):1688-1700. doi:10.1016/S1470-2045(17)30717-9

29. Chan A, Moy B, Mansi J, et al. Final efficacy results of neratinib in HER2-positive hormone receptor-positive early-stage breast cancer from the phase III ExteNET trial. Clin Breast Cancer. 2020;21(1):8091.e7. doi:10.1016/j.clbc.2020.09.014

30. Barcenas CH, Hurvitz SA, Di Palma JA, et al. Improved tolerability of neratinib in patients with HER2-positive early-stage breast cancer: the CONTROL trial. Ann Oncol. 2020;31(9):1223-1230. doi:10.1016/j.annonc.2020.05.012

31. Tsukada Y, Fouad A, Pickren JW, Lane WW. Central nervous system metastasis from breast carcinoma. Autopsy study. Cancer. 1983;52 (12):2349-2354. doi:10.1002/1097-0142(19831215)52:12<2349: AID-CNCR2820521231>3.0.CO;2-B

32. Evans AJ, James JJ, Cornford EJ, et al. Brain metastases from breast cancer: identification of a high-risk group. Clin Oncol. 2004;16 (5):345-349. doi:10.1016/j.clon.2004.03.012

33. Stemmler HJ, Kahlert S, Siekiera W, Untch M, Heinrich B, Heinemann V. Characteristics of patients with brain metastases receiving trastuzumab for HER2 overexpressing metastatic breast cancer. Breast. 2006;15 (2):219-225. doi:10.1016/j.breast.2005.04.017

34. Brufsky AM, Mayer M, Rugo HS, et al. Central nervous system metastases in patients with HER2-positive metastatic breast cancer: incidence, treatment, and survival in patients from registHER. Clin Cancer Res. 2011;17(14):4834-4843. doi:10.1158/1078-0432.CCR10-2962

35. Clayton AJ, Danson S, Jolly S, et al. Incidence of cerebral metastases in patients treated with trastuzumab for metastatic breast cancer. $\mathrm{Br}$ $J$ Cancer. 2004;91(4):639-643. doi:10.1038/sj.bjc.6601970

36. Soffietti R, Ahluwalia M, Lin N, Rudà R. Management of brain metastases according to molecular subtypes. Nat Rev Neurol. 2020;16(10):557-574. doi:10.1038/s41582-020-0391-x

37. Ramakrishna N, Temin S, Chandarlapaty S, et al. Recommendations on disease management for patients with advanced human epidermal growth factor receptor 2-positive breast cancer and brain metastases: ASCO clinical practice guideline update. J Clin Oncol. 2018;36 (27):2804-2807. doi:10.1200/JCO.2018.79.2713

38. Awada A, Colomer R, Inoue K, et al. Neratinib plus paclitaxel vs trastuzumab plus paclitaxel in previously untreated metastatic ERBB2-Positive breast cancer: the NEfERT-T Randomized Clinical Trial. JAMA Oncol. 2016;2(12):1557-1564. doi:10.1001/ jamaoncol.2016.0237

39. Freedman RA, Gelman RS, Anders CK, et al. TBCRC 022: a Phase II Trial of Neratinib and Capecitabine for patients with human epidermal growth factor receptor 2-positive breast cancer and brain metastases. J Clin Oncol. 2019;37(13):1081-1089. doi:10.1200/ JCO.18.01511
40. Murthy RK, Loi S, Okines A, et al. Tucatinib, Trastuzumab, and Capecitabine for HER2-Positive Metastatic Breast Cancer. $N$ Engl $J$ Med. 2020;382(7):597-609. doi:10.1056/NEJMoa1914609

41. Xu B, Yan M, Ma F, et al. Pyrotinib plus capecitabine versus lapatinib plus capecitabine for the treatment of HER2-positive metastatic breast cancer (PHOEBE): a multicentre, open-label, randomised, controlled, phase 3 trial. Lancet Oncol. 2021;22(3):351-360. doi:10.1016/S1470-2045(20)30702-6

42. Rugo HS, Im S-A, Cardoso F, et al. Efficacy of margetuximab vs trastuzumab in patients with pretreated ERBB2-Positive advanced breast cancer: a phase 3 Randomized Clinical Trial. JAMA Oncol. 2021;7(4):573. doi:10.1001/jamaoncol.2020.7932

43. Modi S, Saura C, Yamashita T, et al. Trastuzumab deruxtecan in previously treated HER2-positive breast cancer. $N$ Engl $\mathrm{J}$ Med. 2020;382(7):610-621. doi:10.1056/NEJMoa1914510

44. Banerji U, van Herpen CML, Saura C, et al. Trastuzumab duocarmazine in locally advanced and metastatic solid tumours and HER2-expressing breast cancer: a phase 1 dose-escalation and dose-expansion study. Lancet Oncol. 2019;20(8):1124-1135. doi:10.1016/S1470-2045(19)30328-6

45. Mueller V, Paplomata E, Hamilton EP, et al. 2750 Impact of tucatinib on health-related quality of life (HRQoL) in patients with HER2+ metastatic breast cancer (MBC) with and without brain metastases (BM). Ann Oncol. 2020;31:S349-S350. doi:10.1016/j. annonc.2020.08.377

46. Lin NU, Borges V, Anders C, et al. Intracranial efficacy and survival with tucatinib plus trastuzumab and capecitabine for previously treated HER2-positive breast cancer with brain metastases in the HER2CLIMB Trial. J Clin Oncol. 2020;JCO2000775.

47. Lin NU, Diéras V, Paul D, et al. Multicenter phase II study of lapatinib in patients with brain metastases from HER2-positive breast cancer. Clin Cancer Res. 2009;15(4):1452-1459. doi:10.1158/10780432.CCR-08-1080

48. Bachelot T, Romieu G, Campone M, et al. Lapatinib plus capecitabine in patients with previously untreated brain metastases from HER2-positive metastatic breast cancer (LANDSCAPE): a single-group Phase 2 study. Lancet Oncol. 2013;14(1):64-71. doi:10.1016/S1470-2045(12)70432-1

49. Ramakrishna N, Temin S, Chandarlapaty S, et al. Recommendations on disease management for patients with advanced human epidermal growth factor receptor 2-positive breast cancer and brain metastases: American Society of Clinical Oncology clinical practice guideline. $J$ Clin Oncol. 2014;32(19):2100-2108. doi:10.1200/ JCO.2013.54.0955

50. Cocco E, Javier Carmona F, Razavi P, et al. Neratinib is effective in breast tumors bearing both amplification and mutation of ERBB2 (HER2). Sci Signal. 2018;11(551):551. doi:10.1126/scisignal.aat9773

51. Hyman DM, Piha-Paul SA, Won H, et al. HER kinase inhibition in patients with HER2- and HER3-mutant cancers. Nature. 2018;554 (7691):189-194. doi:10.1038/nature25475

52. Freedman RA, Gelman RS, Wefel JS, et al. Translational Breast Cancer Research Consortium (TBCRC) 022: a Phase II Trial of Neratinib for patients with human epidermal growth factor receptor 2-positive breast cancer and brain metastases. J Clin Oncol. 2016;34 (9):945-952. doi:10.1200/JCO.2015.63.0343

53. Montemurro F, Delaloge S, Barrios CH, et al. Trastuzumab emtansine (T-DM1) in patients with HER2-positive metastatic breast cancer and brain metastases: exploratory final analysis of cohort 1 from KAMILLA, a single-arm phase IIIb clinical trial. Ann Oncol. 2020;31(10):1350-1358. doi:10.1016/j.annonc.2020.06.020

54. Jerusalem G, Park YH, Yamashita T, et al. $138 \mathrm{O}$ CNS metastases in HER2-positive metastatic breast cancer treated with trastuzumab deruxtecan: DESTINY-Breast01 subgroup analyses. Ann Oncol. 2020;31:S63-S64. doi:10.1016/j.annonc.2020.03.239 


\section{Publish your work in this journal}

Drug Design, Development and Therapy is an international, peerreviewed open-access journal that spans the spectrum of drug design and development through to clinical applications. Clinical outcomes, patient safety, and programs for the development and effective, safe, and sustained use of medicines are a feature of the journal, which has also been accepted for indexing on PubMed Central. The manuscript management system is completely online and includes a very quick and fair peer-review system, which is all easy to use. Visit http://www. dovepress.com/testimonials.php to read real quotes from published authors. 